\title{
Comparison between traditional and electronic ETDRS charts
}

\author{
Claudio Campa ( \\ Ophthalmic Surgery Unit, University Hospital of Ferarra, Cona, Ferrara, Italy
}

\begin{abstract}
BACKGROUND: The aim of the study was to compare the visual acuity (VA) score obtained in both normal subjects and patients with different eye diseases by using TOPCON CP-22 electronic ETDRS charts (i.e. E-ETDRS) and standard ETDRS charts (S-ETDRS).

MATERIAL AND METHODS: The primary outcome of this observational prospective study was the difference in median VA score (in letters) recorded in 60 patients by using both E-ETDRS and S-ETDRS. There were 60 subjects enrolled in the study: 20 normal, 20 with diabetic retinopathy and 20 with age-related macular degeneration.

RESULTS: Median number of letters read was 72.5 S-ETDR and 77 for E-ETDR $(p<0.01)$. A subgroup analysis disclosed that the difference in VA score between the 2 devices was more pronounced $(\mathrm{p}<0.01)$ when considering healthy subjects compared to patients affected by diabetic retinopathy $(\mathrm{p}=0.02)$ or age-related macular degeneration $(\mathrm{p}=0.04)$.
\end{abstract}

CONCLUSIONS: Small but significant discrepancies between the 2 devices have been detected, especially when recording high VA values.

KEY WORDS: visual acuity measurements; electronic ETDRS charts; traditional ETDRS charts; age-related macular degeneration; diabetic retinopathy

Ophthalmol J 2020; Vol. 5, 46-50

\section{INTRODUCTION}

Since their first introduction more than 30 years ago [1], ETDRS charts have represented always the gold standard for visual acuity measurement. These charts are based on the Bailey-Lovie principles: letters of approximately equal legibility, equal letters per line and a regular progression in letter size between lines. These features assure many advantages compared to the most popular Snellen charts: visual acuity (VA) measurement is always accurate regardless of initial vision; test-retest variability (TRV) is low, thus increasing the ability to detect subtle change in vision; acuity score can be easily used in statistical analysis. In the era of anti-VEGF therapy for many retinal diseases, where improvement of visual acuity is common, the ability to measure even small changes in vision has become an important tool to determine the response to treatment. This explains why the ETDRS charts are being used more and more often not only in clinical trials but also in routine clinical practice.

The original ETDRS charts are printed on opaque, washable polystyrene and mounted on an illuminator cabinet which uses fluorescent tubes which should be replaced annually and "burned in" (i.e., left on continuously for 96 hours) before using the ETDRS light box (Fig. 1A).

Recently many companies have launched into the market LCD screens with both Snellen and ETDRS charts. These devices are very convenient 


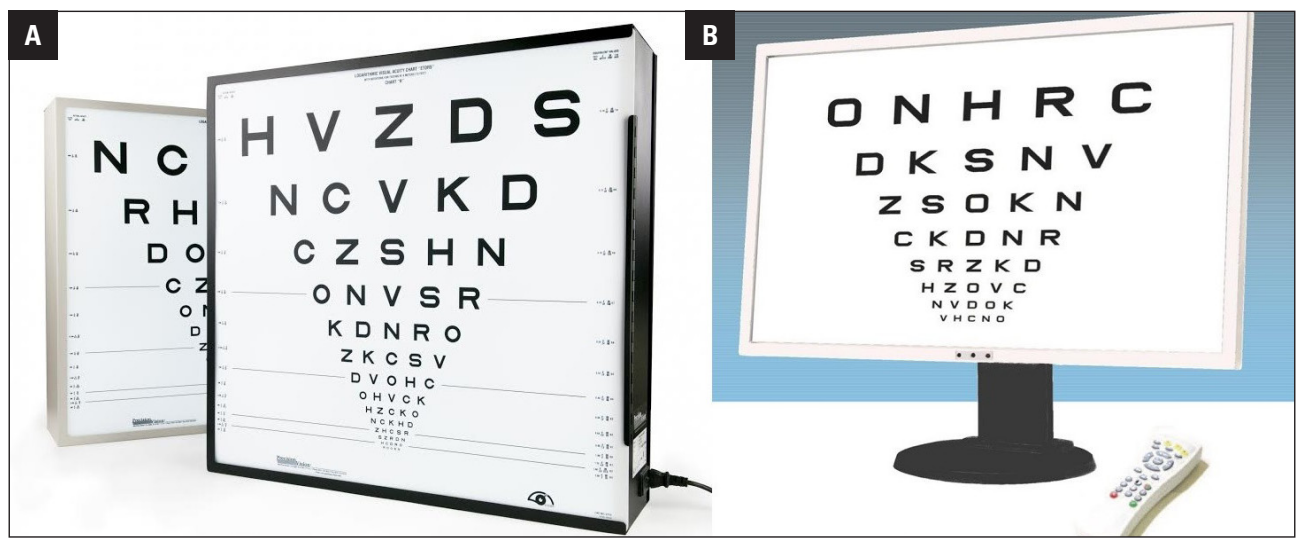

FIGURE 1. A. Original ETDRS chart (S-ETDRS). B. Electronic Topcon CP-22 (E-ETDRS)

because they do not require any maintenance, allow a quickly switch form one test to another and can be used at different distances.

Unfortunately most of them have not been validated in clinical studies, so it is difficult to assume that visual acuity recorded on LCD screen ETDRS charts is equivalent to that performed with standard ETDRS charts.

Topcon CP-22 is (Topcon, Japan) is a led LCD system which incorporates all important visual acuity and colour vision tests. It has a 22 " wide screen with high resolution, high contrast and a high brightness (Fig. 1B).

Aim of this study was to compare the VA score obtained in both normal subjects and patients with different eye diseases by using CP-22 (i.e. electronic ETDRS charts E-ETDRS) and standard ETDRS charts (S-ETDRS).

\section{MATERIAL AND METHODS}

This was an observational single centre study. The primary outcome of the study was the change in median visual acuity score recorded between EETDRS and S-ETDRS. We decided to use the median for the primary outcome because it is better suited for skewed distributions to derive at central tendency since it is much more robust and sensible. For sample size calculation we considered significant a difference of 6 ETDRS letters, since it has been demonstrated that the test-retest reliability falls within 5 letters for the majority of patients for both methods ( $89 \%$ and $87 \%$ for E- and S-ETDRS, respectively) [2]. With an estimated standard deviation of the difference of 0.05 [3], a value for alpha of 0.01 and a power of the test of 0.9 , the required number of patients when using a two-tailed t-test was 5.

Patients enrolled in the study were divided into 3 groups of 20 subjects each: group A, B and C. Only one eye (right) was investigated. The research was performed in compliance with the guidelines of the Declaration of Helsinki. An informed consent was obtained from the subjects after explanation of the nature and possible consequences of the study.

Inclusion criteria for all groups were age $>18$ years and visual acuity better than $2.00 \mathrm{log}$ MAR in the study eye. Additional inclusion criteria were diabetic retinopathy (any grade, with or without maculopathy) for group B and age related macular degeneration (dry or exudative) for group $\mathrm{C}$, respectively. Exclusion criterion for all groups was the inability to sign an informed consent. Additional exclusion criteria were: for group A any ocular disease except refractive errors; for group B ocular diseases different from diabetic retinopathy; for group $\mathrm{C}$ ocular diseases different from age related macular degeneration. Each subject enrolled in the study underwent two visits performed by the same examiner (C.C.) in two different days, 7-10 days a part. During the first visit the study eye was refracted on ETDRS chart R (No. 2110, Precision Vision, La Salle, Illinois). Once obtained the optimal refractive correction, best corrected visual acuity was measured on E-ETDRS and $S$-ETDRS, in random order chosen by flipping a coin (simple randomization).

During examination room illumination was kept constant and before starting each test luminance in the centre of VA chart was checked in order to avoid values $>161.4$ lux (with retroillumination/monitor 
off). For S-ETDRS we used chart 1 (No. 2111, Precision Vision, La Salle, Illinois) at 4 meters. For E-ETDRS the monitor of CP-22 was placed at 2 meters, according to manufacture instructions. When the subject could not see at least 20 letters at these distances then both charts were placed at 1 meter and +0.50 sphere was added to the refraction.

With both charts we used the same VA line presentation testing procedure, which has been widely described in the TAP study visual acuity protocol [4].

At the second visit the VA of study eye was re-tested on both chart (chosen with a random order) using the refraction determined at the previous visit. Patients experiencing any visual symptoms in the interval between the 2 visits had to exit the study.

Origin Pro 8 (Origin Lb Corporation) was used for all the statistical analyses. After analysing data distribution with Shapiro-Wilk test, either Wilcoxon or paired t-test was used for comparisons.

\section{RESULTS}

Sixty subjects completed the study. Demographic and baseline characteristics are shown in Table 1 . When considering the whole study popula- tion the median number of letters read at the first visit on S-ETDRS and E-ETDR was 77 and 72.5, respectively $(\mathrm{p}<0.01)$. Analysing in details the VA score in each group (Fig. 2), mean number of letters read with S-ETDRS and E-ETDRS was 90.2 and 83.2 in group $A(p<0.01), 68.7$ and $66.4(p=0.02)$ in group $\mathrm{B}$, and 66.5 letters and 62 on E-ETDRS $(\mathrm{p}=0.04)$ in group $\mathrm{C}$, respectively.

With the second visit we evaluated the test-retest reliability of both charts. As shown in Figure 3 there was a significant difference $(\mathrm{p}<0.05)$ between the two devices, with a mean difference of 0.75 letters for E-ETDRS and 2.81 for S-ETDRS.

\section{DISCUSSION}

With this study we have demonstrated that small but significant differences between S-ETDR and E-ETDRS (namely TOPCON CP-24) do exist. When considering the whole group of subjects investigated, in fact we found a mean discrepancy of 5.5 letters between the two devices. This disparity was even more pronounced when taking into considerations the group with the highest VA (i.e. healthy subjects), with a mean difference of 7 letters. These differences are not only statistically but also clinically significant: indeed a change of 5 or

Table 1. Demographic and baseline characteristics of study population

\begin{tabular}{|c|c|c|c|}
\hline & Group A & Group B & Group C \\
\hline Mean age $( \pm S D$ ) (years) & $41( \pm 8.1)$ & $52.5( \pm 4.7)$ & $69.3( \pm 5.2)$ \\
\hline $\operatorname{Sex}(M / F)$ & $12 / 8$ & $12 / 8$ & $10 / 10$ \\
\hline $\begin{array}{l}\text { Mean refractive errors }( \pm S D \text { ) } \\
\text { Spherical equivalent }\end{array}$ & $-1.12( \pm 0.3)$ & $-1.07( \pm 0.5)$ & $-0.73( \pm 0.2)$ \\
\hline
\end{tabular}

SD — standard deviation

\section{A}

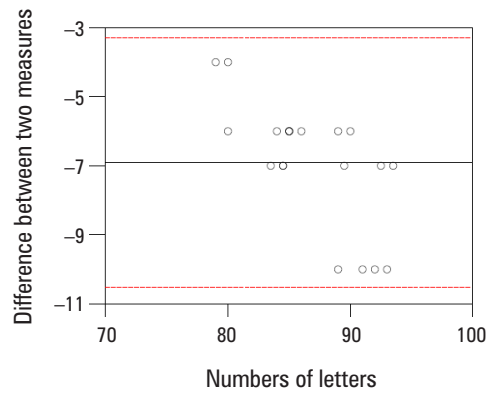

B

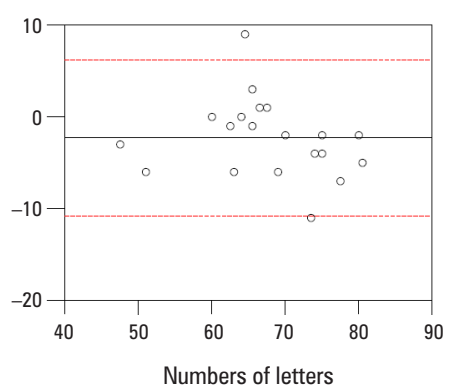

c

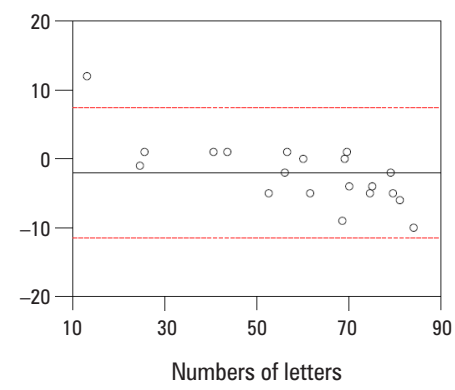

FIGURE 2. Bland-Altmann plots of visual acuity (VA) measurements expressed in letters for group A (A), B (B) and C (C). In the scatter plot the $\mathrm{Y}$ axis shows the difference between the two paired measurements and the $\mathrm{X}$ axis represents the average of these measures. Solid line shows the mean difference, doted lines represent the confidence interval (from $-1.96 \mathrm{~s}$ to $+1.96 \mathrm{~s}$ ) 
A

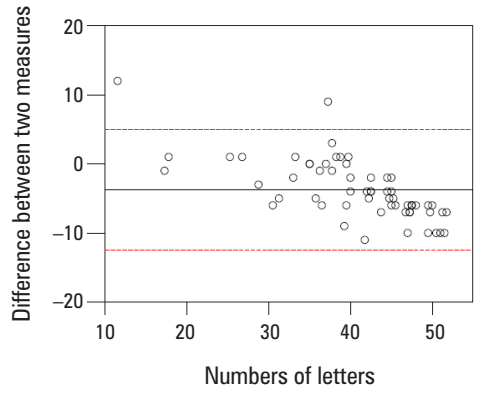

B

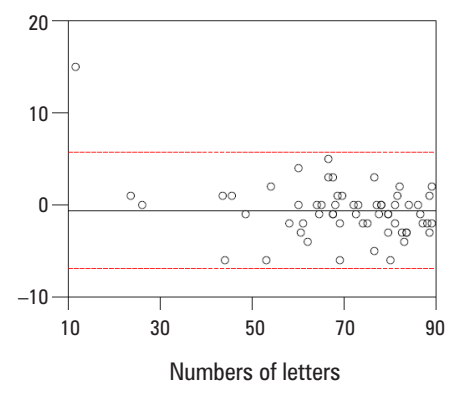

FIGURE 3. Bland-Altmann plots of test-retest reliability expressed in letters for S-ETDRS (A) and E-ETDRS (B). The plot illustrates the agreement between time 1 and time 2 and identifies possible outliers. Reference lines shows mean difference between time 1 and time 2 (solid line), and $95 \%$ limits of agreement for the mean difference (doted lines)

more letters is often used as marker of response to anti-VEGF treatment.

It is difficult to explain this difference, but the most obvious reason may be the limited resolution of the screen. Differences in technical specifications such as calibration of sizes, spacing, luminance and contrast levels between the two devices deserve further investigation.

The only other study [2] we found in literature comparing traditional and electronic ETDRS charts showed similar results, with scores of S-ETDRS and of a computerized vision tester called EVA (electronic visual acuity) differing by $\leq 0.1 \log$ MAR and by $\leq 0.2 \log$ MAR on $74 \%$ and $94 \%$ of patients tested, respectively. In that study the difference was at least in part ascribed to the effect of using single-letter presentations with the E-ETDRS and line presentations with the S-ETDRS [2].

In our study both ETDRS had a line presentation procedure; however, a relevant difference between the 2 charts was that S-ETDRS were used at 4 meters while E-ETDRS at 2 meters (as per indication of the company). This difference could be a reason for the discrepancy in VA we recorded. For instance, Kaiser [5] has demonstrated, by using S-ETDRS charts at 2 and 4 meters, a statistically significant difference in the VA measurements at the two distances. Moreover other authors [6] have found an improvement in visual acuity as observation distance is reduced from six meters to approximately three meters, followed by a decrease in acuity as the distance is further reduced to 0.75 meter.

Both S-ETDRS and E-ETDRS in our study showed high test-retest reliability across the range of VA, with a slight lower reliability for S-ETDRS. Several other studies have demonstrated analogues results with ETDRS testing and similar optotype testing with letter scoring $[1,7-9]$.

\section{CONCLUSIONS}

In summary, the E-ETDRS provided by TOPCON CP-24 has high test-retest reliability and good concordance with S-ETDRS testing, at least for medium and low VA. However, since small discrepancies between the 2 devices have been detected, especially at highest VA, caution must be taken if the 2 devices are used interchangeably during the follow-up of patients undergone a particular ocular treatment. Future work is indicated to determine whether the E-ETDRS testing algorithm can be modified to further reduce the above mentioned letter discrepancy with S-ETDRS.

\section{Conflict of interest and funding}

None.

\section{REFERENCES}

1. Ferris F, Kassoff A, Bresnick G, et al. New Visual Acuity Charts for Clinical Research. Am J Ophthalmol. 1982; 94(1): 91-96, doi: 10.1016/0002-9394(82)90197-0.

2. Beck RW, Moke PS, Turpin AH, et al. A computerized method of visual acuity testing: adaptation of the early treatment of diabetic retinopathy study testing protocol. Am J Ophthalmol. 2003; 135(2): 194-205, doi: 10.1016/s0002-9394(02)01825-1, indexed in Pubmed: 12566024.

3. Rosser DA, Murdoch IE, Cousens SN. The effect of optical defocus on the test-retest variability of visual acuity measurements. Invest Ophthalmol Vis Sci. 2004; 45(4): 1076-1079, doi: 10.1167/iovs.031320, indexed in Pubmed: 15037571.

4. Photodynamic therapy of subfoveal choroidal neovascularization in age-related macular degeneration with verteporfin: one-year results of 2 randomized clinical trials - TAP report. Treatment of 
age-related macular degeneration with photodynamic therapy (TAP) Study Group. Arch Ophthalmol. 1999; 117(10): 1329, doi: 10.1001/ archopht.117.10.1329, indexed in Pubmed: 10532441.

5. Kaiser PK. Prospective evaluation of visual acuity assessment: a comparison of snellen versus ETDRS charts in clinical practice (An AOS Thesis). Trans Am Ophthalmol Soc. 2009; 107: 311-324, indexed in Pubmed: 20126505

6. Geddes M, McLean J, McMonnies C, et al. The variation of visual acuity with observation distance. Austral J Optometry. 1966; 49(6): 164-9, doi: 10.1111/j.1444-0938.1966.tb04527.x.
7. Lovie-Kitchin JE. Validity and reliability of visual acuity measurements. Ophthalmic Physiol Opt. 1988; 8(4): 363-370, doi: 10.1111/j.14751313.1988.tb01170.x, indexed in Pubmed: 3253626.

8. Arditi A, Cagenello R. On the statistical reliability of letter-chart visual acuity measurements. Invest Ophthalmol Vis Sci. 1993; 34(1): 120-129, indexed in Pubmed: 8425819.

9. Blackhurst DW, Maguire MG. Reproducibility of refraction and visual acuity measurement under a standard protocol. The Macular Photocoagulation Study Group. Retina. 1989; 9(3): 163-169, indexed in Pubmed: 2480626. 